\title{
Airborne Electronic Equipment Health Condition Assessment Technique
}

\author{
Ai-Qiang XU ${ }^{1, a}$, Lei MENG ${ }^{2, b}$, Jing-Hua ZHU ${ }^{3, c}$ \\ ${ }^{1}$ Airborne Vehicle Engineering Department; Naval Aeronautical Engineering University, Yantai, \\ China \\ ${ }^{2}$ Airborne Vehicle Engineering Department; Naval Aeronautical Engineering University, Yantai, \\ China \\ ${ }^{3}$ 92212force, Qingdao, China

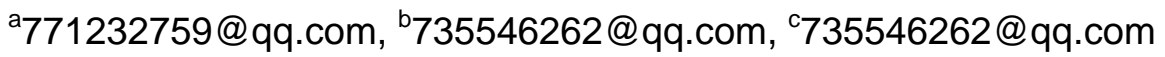 \\ ${ }^{*}$ Corresponding author
}

Keywords: Airborne Electronic Equipment, Parameter Estimate, Clustering by Fast Search and Find of Density Peaks, Particle Swarm Optimization Extreme Learning Machine.

\begin{abstract}
Airborne Electronic Equipment data is unmeasurable, and computing the parameter based on component model is inaccurate and is time costing. The paper presents airborne electronic equipment parameter estimate method based on clustering by fast search and find of density peaks (CFSFDP) and ant colony optimization extreme learning machine (ACO-ELM). Firstly, the CFSFDP method was utilized to cluster the test bench data in the whole behavior range, and then, a sub-estimator was designed in each cluster using ACO-ELM. In the process of designing the sub-estimator with ACO-ELM, the particle swarm optimization algorithm was utilized to search the best hidden nodes number of extreme learning machine for getting the best topological structure.
\end{abstract}

\section{Introduction}

The parameter of Airborne electronic equipment's test is an important indicator for the state evaluation and health management. However, there are still some problems that cannot be measured in the health state evaluation of airborne electronic equipment ${ }^{[1]}$. Airborne electronic equipment health management system can use the stability of the estimated parameters of the value of the overall performance of the state assessment and fault diagnosis ${ }^{[2]}$.Accurate airborne electronic equipment models need to establish based on the model of the method, usually by Kalman filter contains control thrust system and component health parameters and u6nmeasured parameters, can be estimated parameters is likely to be limited in the number of measurable parameters, although under underdetermined condition ${ }^{[3]}$, by singular value decomposition can to a certain extent, to solve the problem, but the complexity of the modeling, model to solve the poor real-time ${ }^{[4]}$ and solving of airborne electronic equipment model ${ }^{[5]}$ usually cannot be separated from parameter measurement. Based on methods of data directly using the measured parameters, mapping specific parameters and thrust, people use based on neural network, least square support vector machine ${ }^{[9,10]}$ design of the parameter estimator, the theory has made good results. Comprehensive domestic and foreign research status in parameter estimation are three key research, one is the selection of the input parameters, the mechanism analysis, correlation analysis ${ }^{[6]}$, cross validation leave a method ${ }^{[7]}$ and nuclear induced to a higher dimensional space ${ }^{[9]}$ method is extracted from the input feature data; the second is airborne electronic state division, usually according to the height and Mach number directly into the packet line ${ }^{[10]}$, K-means clustering ${ }^{[1]}$ and other clustering methods, parameter estimation method for mapping selection, mainly uses the neural network (radial basis, BP network, extreme learning machine), support vector machine (SVM) and least squares support vector machine (SVM) etc.

This paper puts forward the parameters based on the search for density extremum clustering (clustering by fast search and find of the density peaks, CFSFDP) ${ }^{[12]}$ and particle swarm limit learning (particle swarm optimization extreme learning machine, PSO-ELM) method ${ }^{[13]}$ estimation. CFSFDP $^{2}$ 
is a new clustering method published on Science in June 2014. ly easy to produce error clustering results and an unknown number of clustering, while the use of CFSFDP can solve this problem. $\mathrm{ELM}^{[13]}$ is in the field of machine learning has good ability in classification and regression of single hidden layer feed forward neural networks, with parameter setting is simple, high intelligent degree, advantages of computational speed ${ }^{[14]}$, because the network topology is affect the accuracy and real-time calculation ability important factors ${ }^{[15]}$.

\section{ACO-ELM}

\section{ELM}

The section headings are in boldface capital and lowercase letters. Second level headings are typed as part of the succeeding paragraph (like the subsection heading of this paragraph). ELM is a single hidden layer feed forward neural network, for any number of different samples $\left(x_{i}, y_{i}\right)$, $\boldsymbol{x}_{i}=\left[x_{i 1}, x_{i 2}, \ldots, x_{i n}\right]^{\mathrm{T}} \in \boldsymbol{R}^{n}, \boldsymbol{y}_{i}=\left[y_{i 1}, y_{i 2}, \ldots, y_{i m}\right]^{\mathrm{T}} \in \mathrm{R}^{\mathrm{m}}$ and has $\tilde{N}$ mathematical model of hidden layer nodes, activation function $g(x)$ is as follows. $\sum_{i=1}^{\tilde{N}} \boldsymbol{\beta}_{i} \boldsymbol{g}_{i}\left(\mathrm{x}_{j}\right)=\sum_{i=1}^{\tilde{N}} \boldsymbol{\beta}_{i} \boldsymbol{g}_{i}\left(\boldsymbol{w}_{i} \cdot \boldsymbol{x}_{j}+b_{i}\right)=\mathrm{o}_{j}$

Type: $\boldsymbol{w}_{i}=\left[\boldsymbol{w}_{i 1}, \boldsymbol{w}_{i 2}, \ldots, \boldsymbol{w}_{i n}\right]^{\mathrm{T}}$ is connected to the it hidden layer nodes and nodes in the input weight vector, $\boldsymbol{\beta}_{i}=\left[\beta_{i 1}, \beta_{i 2}, \ldots, \beta_{i m}\right]^{\mathrm{T}}$ for the number of nodes in the hidden layer is connected to the hidden layer nodes and output layer node weight vectors, ${ }^{b_{i}}$ the hidden layer node threshold, ${ }^{\boldsymbol{w}_{i} \cdot \boldsymbol{x}_{j}}$ is the inner product of the two. The linear combination of the output layer nodes is usually selected.

$$
\begin{aligned}
& \sum_{i=1}^{\tilde{N}} \boldsymbol{\beta}_{i} \boldsymbol{g}_{i}\left(\boldsymbol{w}_{\boldsymbol{i}} \cdot \boldsymbol{x}_{j}+b_{i}\right)=y_{j}, j=1,2, \ldots, N . \\
& H \boldsymbol{\beta}=\boldsymbol{Y} \\
& \boldsymbol{H}=\left[\begin{array}{ccc}
g\left(\boldsymbol{w}_{\boldsymbol{1}} \cdot \boldsymbol{x}_{1}+b_{1}\right) \cdots g\left(\boldsymbol{w}_{\tilde{N}} \cdot \boldsymbol{x}_{1}+b_{\tilde{N}}\right) \\
\vdots & \cdots & \vdots \\
g\left(\boldsymbol{w}_{\boldsymbol{l}} \cdot \boldsymbol{x}_{N}+b_{1}\right) \cdots g\left(\boldsymbol{w}_{\tilde{N}} \cdot \boldsymbol{x}_{N}+b_{\tilde{N}}\right)
\end{array}\right]_{N \times \tilde{N}} \quad \boldsymbol{\beta}=\left[\begin{array}{l}
\boldsymbol{\beta}_{\boldsymbol{1}}^{T} \\
\vdots \\
\boldsymbol{\beta}_{\tilde{N}}^{T}
\end{array}\right]_{\tilde{N} \times m} \quad \boldsymbol{Y}=\left[\begin{array}{l}
\boldsymbol{y}_{\boldsymbol{1}}^{\boldsymbol{T}} \\
\vdots \\
\boldsymbol{y}_{N}^{\boldsymbol{T}}
\end{array}\right]_{N} ?
\end{aligned}
$$

Further, given a small positive ${ }^{\varepsilon}, \tilde{N} \leq N$ randomly selected $\boldsymbol{w}_{i}, b_{i}, \boldsymbol{w}_{\boldsymbol{i}} \in \mathrm{R}^{n}, b_{i} \in \mathrm{R}$, which makes the error $\left\|\boldsymbol{H}_{N \times \tilde{N}} \boldsymbol{\beta}_{\tilde{N} \times m}-\boldsymbol{Y}_{N \times m}\right\|<\varepsilon$. The proof of literature ${ }^{[13],}$ on the basis of the theory, proposed the method of extreme learning machine, network training process is equivalent to the process of finding the least squares solution of linear system $\boldsymbol{H} \boldsymbol{\beta}=\boldsymbol{Y}$. Generalized inverse matrix for Moore $\widehat{\boldsymbol{\beta}}=\boldsymbol{H}^{\dot{\boldsymbol{Y}} \boldsymbol{Y}}$ - Penrose. $\boldsymbol{H}^{\dagger}$ is the generalized inverse matrix of $\boldsymbol{H}$.

\section{ACO-ELM}

In the standard ELM randomly selected weight vector ${ }^{w_{i}}$ and threshold ${ }^{b_{i}}$, is necessary to determine the number of hidden layer neurons $\tilde{N}$, but study found, selections ${ }^{w_{i}}$ and ${ }^{b_{i}}$ still has a certain degree of influence the results, so the design of particle swarm optimization strategy determined according to the parameter estimation problem of optimal $\tilde{N}$ algorithm is shown in Figure 1. 


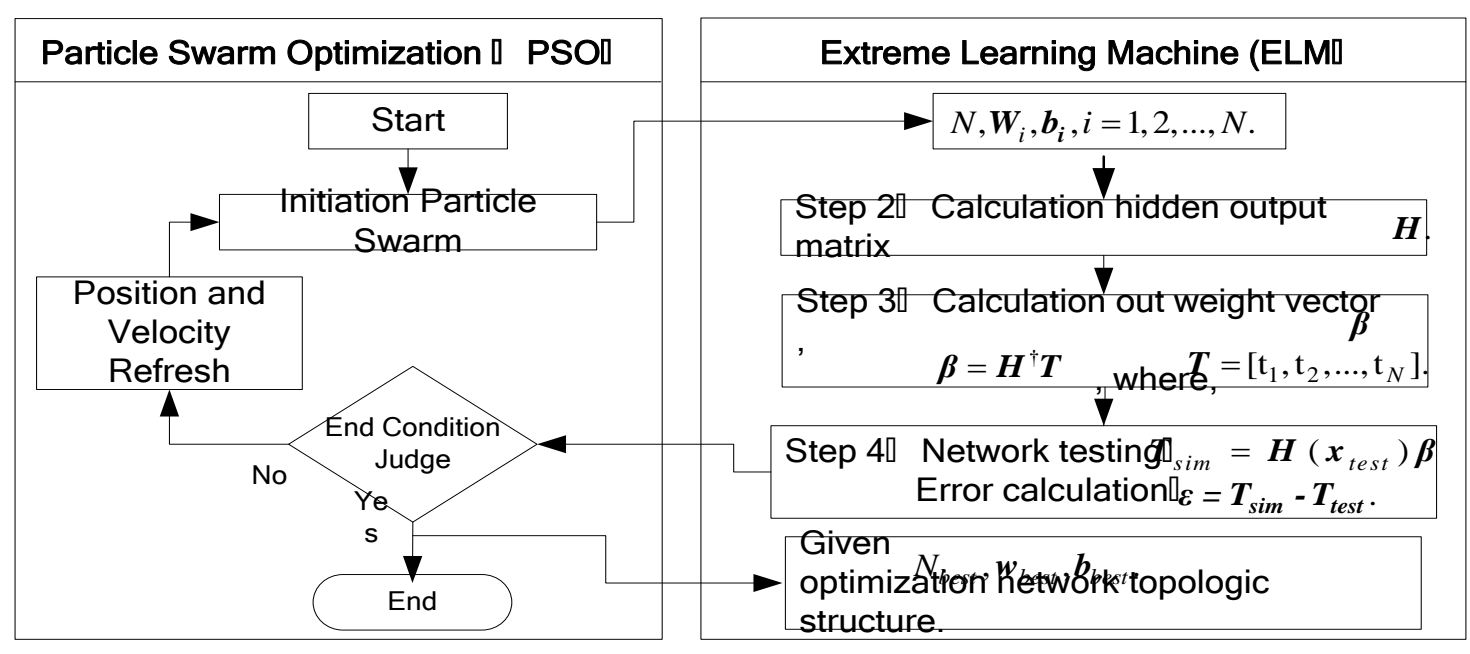

Fig.1 ACO-ELM Algorithm Scheme

Among them, the fitness function is set to ELM estimated the relative error between the parameter values and the actual values:

$$
p_{i j}^{k}(t)= \begin{cases}\frac{\tau_{i j}^{\alpha}(t) \eta_{i j}^{\beta}(t)}{\sum_{v \in S} \tau_{i v}^{\alpha}(t) \eta_{i v}^{\beta}(t)}, & j \in S \\ 0, & j \notin S\end{cases}
$$

Type: ${ }^{\tau_{i j}(t)}$ is ${ }^{t}$ moment side arc ${ }^{(i, j)}$ trajectory of strength, ${ }^{(i, j)}$ residual amount of information and $\tau_{i j}(0)=c, i, j=1,2, \ldots, n ; i \neq j, \quad \eta_{i j}(t)$ is a ${ }^{(i, j)}$ constant, constantly side arc of visibility, reflecting position ${ }^{i}$ shifts to ${ }^{j}$ of the desired strength, $\alpha$ is the trajectory of relative importance, $\beta$ is the relative importance of visibility, $s$ is the feasible point set.

The track strength update equation for adjusting the amount of information is:

$$
\begin{aligned}
& \tau_{i j}(t+1)=\rho \tau_{i j}(t)+\Delta \tau_{i j}, \rho \in(0,1) \\
& \Delta \tau_{i j}=\sum_{k=1}^{m} \Delta \tau_{i j}^{k}
\end{aligned}
$$

$\Delta \tau_{i j}^{k}$ is the ${ }^{k}$ ants in the cycle to stay on the path ${ }^{(i, j)}$ information, ${ }^{\Delta \tau_{i j}}$ the middle circular diameter on the amount of information increment; parameters ${ }^{\rho}$ is persistence trajectory; ${ }^{1-} \rho$ is trajectory attenuation degree, said information about the magnitude of the die.

\section{Test Parameter Estimation}

\section{Test Parameters Extraction}

There is a great correlation and coupling between engine parameters, and the suitable feature selection method is very important. There are a total of 38 test parameters of the engine bench, including gas path parameters 25 . Selection and thrust of the correlation coefficient greater than 0.6 above parameters, simultaneous removal of parametric pair wise between correlation coefficients greater than 0.85 and thrust the less relevant parameters, selected from, conditions and thrust related characteristic parameters of a total of 7 , see Table 1. 
Tab.1 Characteristic Parameter Scheme

\begin{tabular}{cc}
\hline Measurement parameters & Symbol \\
\hline Fuel flow & $W_{f}$ \\
Inlet total pressure & $P_{0}^{*}$ \\
High pressure corrected & $N_{2 c o r}$ \\
rotational speed & $P_{3}^{*}$ \\
Burner entrance total pressure & $P_{4}^{*}$ \\
LPT outlet total pressure & $T_{4}^{*}$ \\
LPT outlet total temperature & $D_{p c}$ \\
Throat cross diametrical &
\end{tabular}

\section{CFSFDP Clustering Algorithm}

CFSFDP algorithm theoretical basis for the center of the cluster is surrounded by a small local density, and the local density of these centers is far more than their large point. Each point ${ }^{\boldsymbol{x}_{i}}$ has two characteristic parameters, one is the density $\rho_{i}$; the two is the current point ${ }^{x_{i}}$ and the density is higher than the current point of the distance ${ }^{\delta_{i}}$. CFSFDP algorithm is as follows.

For a data $N$ set $\left\{\boldsymbol{x}_{i}, i=1,2, \ldots \mathrm{N}\right\}$.

Step 1: calculate all point ${ }^{\boldsymbol{x}_{i}}$ features $\left(\rho_{i}, \delta_{i}\right)$.

1) The distance from the point ${ }^{x_{i}}$ to all other points can be used to select all the norm of the distance $d_{i j}=\left\|\boldsymbol{x}_{i}-\boldsymbol{x}_{j}\right\|$;

2) Select the truncation distance ${ }^{d_{c}} ;{ }^{c}=\left\lfloor k^{*} 0.5^{*} n \times(n-1)\right\rfloor, d_{c}=\boldsymbol{d}_{\text {all }}(\mathrm{c}) ;\lfloor\rfloor$ is for four to five homes into the principle of integer, type: $k$ is truncation distance ratio, usually $1-2 \%, \boldsymbol{d}_{\text {all }}$ take for values in ascending order of all distances;

3) Calculate the density ${ }^{\rho_{i}}$ of the point ${ }^{x_{i}}$. The density can be calculated according to the truncated distance, and the density can also be calculated according to the Gauss kernel density;

$$
\begin{aligned}
& \rho_{i}=\sum_{j} \chi\left(d_{i j}-d_{c}\right) \\
& \chi(\mathrm{x})= \begin{cases}1 & x<0 \\
0 & \text { otherwise }\end{cases}
\end{aligned}
$$

4) Gets $\delta_{i},{ }_{i}$ defines $\boldsymbol{x}_{i}$ the distance to the higher density point.

$$
\delta_{i}= \begin{cases}\max _{j}\left(d_{i j}\right) & \rho_{i}=\max \left(\rho_{i}\right) \\ \min _{j: \rho_{j}>\rho_{i}}\left(d_{i j}\right) & \text { otherwise }\end{cases}
$$

Step2: cluster center.

1) To draw the global decision tree, that is, drawing the $\delta-\rho$ drawing;

2) Select an unusually large number of points $\rho$ and $\delta$ as the cluster center $\boldsymbol{x}_{\text {center }}=\left(\boldsymbol{x}_{j}\right), j=1,2 \ldots K$.

Step3: clustering, find core set and set halo.

In accordance with the density of all data points from the large to small array, the point ${ }^{x_{i}}$ due to the density of the point ${ }^{x_{i}}$ of concentration and its distance from the nearest point of the class.

Collecting the idle state above the bench test data, a total of 9971 group, the truncated kernel density CFSFDP clustering of decision tree effect diagram as shown in Figure 2, according to the clustering center selection principle, clustering center of the engine bench test data and other data 
points have very clear boundaries, figure in larger and smaller point was identified as outliers can from the figure clearly defined five clustering centers.

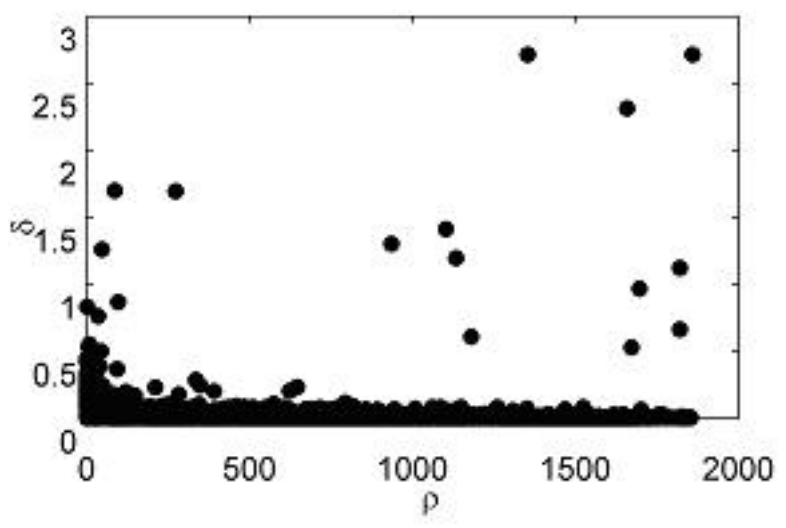

Fig.2 Sample Decision Graph

\section{Test Analysis and Verification}

Normalized thrust parameters, divided according to the working state, a certain type of engine according to the design of slow, cruise, maximum, small afterburner, full afterburner state corresponding thrust respectively $0.02,0.28-0.36 、 0.6136$ and $0.6760,1.00$, which work with afterburner after burner according to the switch signal distinguish. Using ACO-ELM algorithm, the use of optimized and determined, for 124, after the test (10000 tests), and the best choice of the function of the incentive function. Selected with a engine bench test data to establish a thrust estimator model and setting of the sub class in the training data and test data the proportion of 9:1, to verify the validity and the classical RBF neural network, this paper compared, thrust estimation relative error and prediction time results are shown in Table 2.

Tab.2 Sub-thrust Estimator Relative Error Analysis

\begin{tabular}{ccccccccc}
\hline \multirow{2}{*}{ Subclass } & Style & $\begin{array}{c}\text { MAX } \\
/ 10^{-3}\end{array}$ & $\begin{array}{c}\text { Midumn } \\
/ 10^{-3}\end{array}$ & $\begin{array}{c}\text { Min } \\
/ 10^{-5}\end{array}$ & $\begin{array}{c}\text { Mean } \\
/ 10^{-3}\end{array}$ & $\begin{array}{c}\text { STD } \\
/ 10^{-3}\end{array}$ & $\begin{array}{c}\text { Train time } \\
/ \mathrm{ms}\end{array}$ & $\begin{array}{c}\text { Test time } \\
/ \mathrm{ms}\end{array}$ \\
\hline$\# 1$ & ELM & 2.79 & 1.63 & 25.13 & 1.54 & 0.45 & 123.56 & 0.03 \\
& RBF & 4.45 & 1.23 & 6.06 & 1.72 & 1.26 & 1293.31 & 85.00 \\
$\# 2$ & ELM & 3.15 & 0.82 & 9.36 & 1.14 & 0.75 & 59.56 & 0.01 \\
& RBF & 6.96 & 0.36 & 31.16 & 1.25 & 1.78 & 162.31 & 21.10 \\
$\# 3$ & ELM & 2.76 & 0.68 & 23.36 & 1.70 & 0.46 & 59.35 & 0.02 \\
$\# 4$ & RBF & 3.78 & 1.31 & 44.71 & 2.06 & 0.94 & 136.36 & 18.10 \\
& ELM & 2.45 & 0.24 & 23.21 & 0.78 & 0.49 & 223.48 & 0.03 \\
$\# 5$ & RBF & 3.70 & 1.75 & 26.24 & 0.81 & 0.83 & 1235.7 & 16.63 \\
& ELM & 2.63 & 0.83 & 4.45 & 1.12 & 0.60 & 46.25 & 0.04 \\
& RBF & 3.39 & 0.86 & 0.35 & 1.24 & 0.71 & 1363.17 & 29.15 \\
\hline
\end{tabular}

ACO-ELM network of the input and output structure consistent with that RBF input for 7 nodes and output for a node, the RBF neural network in the relative error of the subclass \#1, \#2, \#4, \#5 thrust estimation mean values are less than $2 \%$, but the relative error in the subclass \#3 value to 3.6 per 1000 , although the average value can be found to meet the requirements, but its prediction time beyond the requirements of $2 \mathrm{~ms}$. 


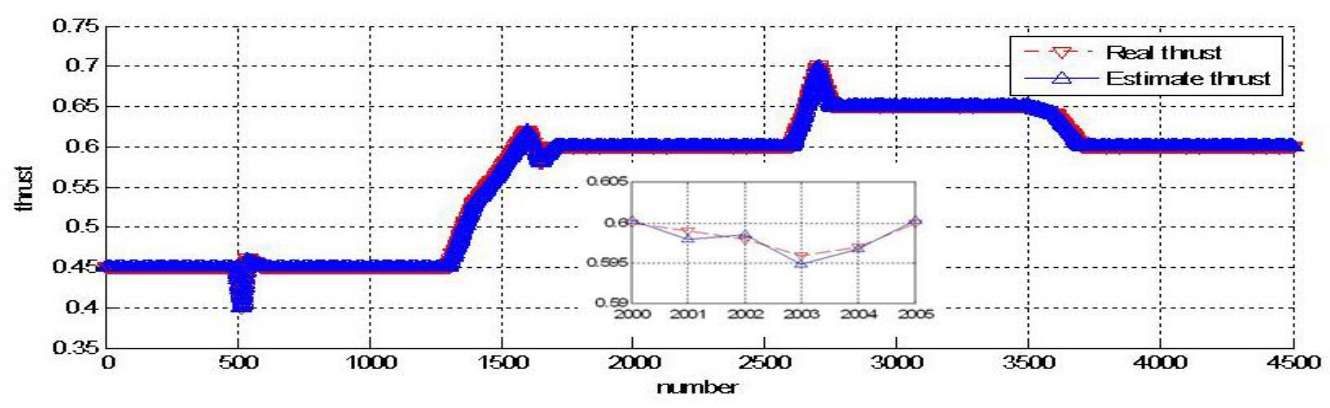

Fig.3 Real Thrust and Estimate Thrust of \#3

Integrated relative error and prediction time are two indexes, and the ACO-ELM is used to estimate the thrust force. Experiments are under the MATLAB environment, in the engineering application can advance good off-line training require the weight value and bias value. According to the periodic update configuration parameters is determined using time, on-line estimation is equivalent to the process of a product, such data in order to predict the time can also guarantee the same level.

\section{Conclusion}

In this paper, a parameter estimation method based on CFSFDP clustering and CFSFDP is proposed, which is based on ACO-ELM to cluster the engine bench test data, and solves the problem of category judgment in the process of engine thrust estimation. Using ACO algorithm to find the optimal ELM of the random parameters and optimize the network topology, so that the ELM has better nonlinear mapping capability for the thrust estimation problem, and improve the accuracy of the thrust parameter estimator. Test results show that the relative error of the estimated mean maximum thrust of 1.08 per thousand, a single parameter prediction time is less than $0.05 \mathrm{~ms}$.

\section{References}

[1] Yao Yanlong, Sun Jianguo.Adaptive genntic neural network algorithm in the application of thrust estimator is designed[J]. Journal of Propulsion Technology, 2007, 22(10): 1749-1755.

[2] Yao Yan-long, Sun Jian-guo. Aeroengine Direct Thrust Control Based on Neural Network Inverse Control[J]. Journal of Propulsion Technology. 2008, 29(2):249-252.

[3] Liu Yi-nan, Zhang Sheng-xiu, Zhang Chao. Aero Engine Thrust Estimator Design Based on Kernel Method[J]. Journal of Propulsion Technology. 2013, 34(6): 829-835.

[4] Zhao Yongping, Sun Jianguo. Least squares support vector regression machine application in the engine thrust is estimated[J]. Journal of Propulsion Technology, 2009, 24(6): 1421-1426.

[5] Litt Jonathan-S. An Optimal Orthogonal Decomposition Method for Kalman Filter-based Turbofan Engine Thrust Estimation[R]. NASA GT2005-68808, 2005.

[6] Donald L.Simon, Jeffrey B. Armstrong. An Integrated Approach for Aircraft Engine Performance Estimation and Fault Diagnostics[J]. Journal of Engineering for Gas Turbines and Power. 2013. (7). 135.071203.

[7] Li Yabo, Li Qiuhong, Huang Xianghua and so on. Aeroengine performance degradation ease control technology[J]. 2012, 27(4): 931-937.

[8] Allan J. Volponi. Gas Turbine Engine Health Management: Past, Present, and Future trends[J] Journal of Engineering for Gas Turbines and Power. May 2014. 136: 051201. 
[9] Henriksson Mattias, Gronstedt Tomas, Breitholtz Claes. Model-based on-board Turbofan Thrust Estimation[J]. Control Engineering Practice, 2011, 19: 602-610.

[10] Zhao Yongping, Sun Jianguo, Wang Qianyu and so on. Based on the mean clustering and reduction of least squares support vector regression machine thrust estimator is designed[J]. Journal of Propulsion Technology, 2010, 25(5): 1178-1185.

[11] Rodriguez Alex, Laio Alessandro. Clustering by Fast Search and Find of Density Peaks[J]. Science, 2014, 344(6191): 1492-1496.

[12] Guang-bin huang Qin-Yu-Zhu, Chee-Kheong-Siew. Extreme Learning Machine Theory and Applications[J]. Neurocomputing , 2006, 70: 489-501.

[13] Nan-ying liang, Guang-Bin Huang. A Fast and Accurate Online Sequential Learning Algorithm for Feedforward Networks[J]. IEEE Transactions on Neural Networks, 2006, 17(6): 1411-1424.

[14] Deng Wenyu, Zheng Qinghua, ChenLin and so on. The method of neural network speed learning[J].Journal of computer, 2010, 33(2): 279-288.

[15] Yang Yiwen, The system identification method and application of limit of learning[D]. Changsha: University of Hunan, 2013. 